\title{
Landmark-Based Stereo Vision
}

\author{
Giovanni B. Garibotto and Marco Corvi \\ Elsag spa, Via Puccini, 2, 16154 Genova, Italy \\ giovanni.garibotto@elsag.it
}

\begin{abstract}
The paper refers an industrial solution to 3D measurements to be used in security application. The basic assumption of the proposed solution is the visibility of a $3 \mathrm{D}$ reference system in the imaged scene. The corresponding vanishing points are used as stereo point pairs at infinity, to compute the rotation between the two stereo views. To achieve a metric reconstruction, the proposed approach relies on the presence in the scene of an artificial landmark having an orthogonal triangular shape with sides of one meter length.. Reflecting cylinders are positioned at the corners and along the sides at equally spaced distances. They represent the initial stereo correspondence for system calibration. This approach has been implemented in a prototype product for the planar reconstruction in case of car accidents using a digital camera. The software application provides a simple and user-friendly interface to the process. The measure errors are always within $2 \%$ (with a $4 \mathrm{Mp}$ camera).
\end{abstract}

Keywords: Computer Vision, camera calibration, 3D stereovision, perspective invariants.

\section{Introduction}

The paper reports an industrial solution, named LSV, Landmark-based Stereo-Vision, to solve the problem of performing distance measurements between points in 3D scenes using stereo pairs of images. This is the classical objective of photogrammetry, widely studied for building reconstruction and virtual reality rendering applications [1], [2], [3]. Our main objective is the development of effective solutions in the field of security, law-enforcement and police investigation [4]. An example of application is the metrical reconstruction of car accident scenes to speed-up the clearance of the traffic area and allow accurate measures to be performed later in a back-office application.3-D scene reconstruction from an image stereo pair is well studied from a theoretical point of view [5]. Projective stereo geometry is described by the fundamental matrix, and methods for the robust determination of this matrix have been proposed [5] [6]. The affine geometry is obtained by identifying the plane at infinity and this task is greatly simplified when a 3D reference system is detectable from the imaged scene (like building structures or road lines) [7]. Finally, in order to get a metric reconstruction, the unit lengths (in the different directions) are required. Unfortunately most natural scenes lack geometric elements for the identification of the plane at infinity and the unit lengths. Therefore, in order to achieve a metric 
reconstruction, our approach relies on the presence of an artificial landmark that provides such reference features through the use of projective invariants and the corresponding vanishing points. Our selected landmark has a right triangular shape with sides of one meter length. Reflecting cylinders are positioned at the corners and along the sides at equally spaced distances, to simplify automatic location and represent the starting points of the camera calibration process. The known lengths of the landmark sides provide a sufficient metric structure to perform 3D measures from a single view, only for 3D points lying on the landmark plane. To achieve a full metric analysis in the 3D scene it is necessary to work with a stereo pair of images. The proposed LSV system has been implemented as a software application that performs 3D measures from a pair of stereo views acquired with a standard digital camera. The application provides a simple and user-friendly interface to the process. The following section summarizes the basic approach based on the use of the triangular landmark.

\section{Stereo Matching at Infinity: Description of the Approach}

Binocular vision allows the metric reconstruction of the 3-dimensional geometry of a scene. This task consists of finding stereo correspondences between points in the two images, and estimating the 3D position of a point in the world from the disparity of its image points [5]. Therefore the computer vision approach to this task involves two problems, namely the identification of stereo corresponding points and stereo calibration to recover the main geometrical features. The proposed approach solves the first problem using an artificial object (landmark) located in the scene, with features that can be "easily" detected and put in correspondence. Furthermore these features are used also to perform stereo calibration. We assume a pinhole camera model, with no intrinsic distortion nor shear. The optical center (intersection of the optical axis with the image plane) is assumed to be known. The only unknown intrinsic parameter is the focal length $\mathrm{f}$. A point $\boldsymbol{P}$ in $3 \mathrm{D}$ is projected on the image plane $\boldsymbol{p}$ by

$$
\boldsymbol{P}=(\mathrm{X}, \mathrm{Y}, \mathrm{Z}) \quad \Rightarrow \quad \boldsymbol{p}=(\mathrm{fX} / \mathrm{Z}, \mathrm{f} \mathrm{Y} / \mathrm{Z})
$$

where $(X, Y, Z)$ are the $3 D$ point coordinates in the frame of reference centered on the camera, with $\mathrm{Z}$ along the optical axis and $\mathrm{f}$ is the focal length of the camera system.

In a stereo pair the two cameras have different frames of reference. The points in a stereo pair of images are related by

$$
\mathrm{P}_{2}=\mathbf{R} \times \mathrm{P}_{1}+\mathbf{T}
$$

where $\mathbf{R}$ is the rotation matrix from the first frame to the second and $\mathbf{T}$ is the translation vector (position of the first camera in the frame of the second), whose length is the baseline of the stereo configuration. This relation is simplified when the points lie at infinity,

$$
\begin{aligned}
& P_{2}=\mathbf{R} \times P_{1} \\
x_{2}= & f \quad X_{2} / Z_{2}=f\left(R_{x x} x_{1}+R_{x y} y_{1}+R_{x z}\right) /\left(R_{z x} x_{1}+R_{z y} y_{1}+R_{z z}\right) \\
y_{2}= & f \quad Y_{2} / Z_{2}=f\left(R_{y x} x_{1}+R_{y y} y_{1}+R_{y z}\right) /\left(R_{z x} x_{1}+R_{z y} y_{1}+R_{z z}\right)
\end{aligned}
$$


ie, the image points of a point at infinity are related by a homography, namely the homography of the plane at infinity. The coefficients of this homography are the coefficients of the rotation matrix between the two camera frames. Therefore the rotation component of the stereo calibration can be determined by finding the correspondence (in the images) of points on the plane at infinity. Image points on the plane at infinity are called vanishing points because they are the images of the "center" of parallel lines in 3D. From the position of the feature elements of the landmark, vanishing points can be computed using projective invariants. Our landmark is a triangle with feature points positioned equally spaced on the sides, and at the corner. The alignment of the points, along one side, allows the estimation of the corresponding vanishing point $V_{l}$, [9], using the cross ratio invariance

$$
\left[\left(p_{0}-p_{2}\right) /\left(p_{0}-p_{1}\right)\right]\left[\left(p_{1}-V_{1}\right) /\left(p_{2}-V_{1}\right)\right]=2
$$

since $\boldsymbol{p}_{\boldsymbol{I}}$ is placed midway along the side of the landmark, of length $\mathbf{L}$. The same operation is repeated for the other side of the landmark to compute the second vanishing point $\boldsymbol{V}_{2}$.

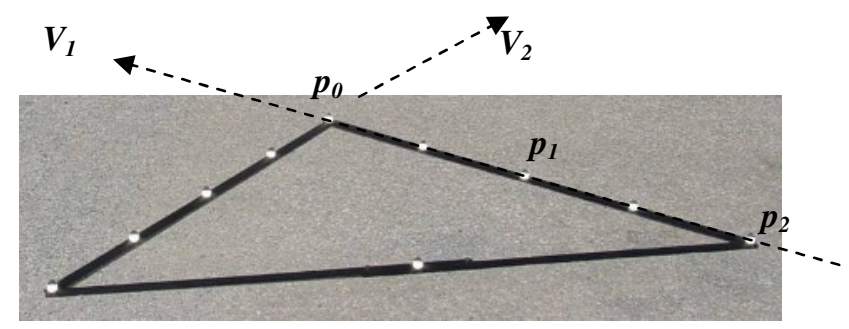

Fig. 1. The triangular landmark is placed into the scene for the automatic detection of the two orthogonal vanishing points $\mathrm{V}_{1}$ and $\mathrm{V}_{2}$

The directions in 3D of these two vanishing points are orthogonal, because of landmark construction, so that:

$$
V_{1} \cdot V_{2}+\mathrm{f}^{2}=0
$$

where $V_{I}$ and $V_{2}$ are the image 2D vectors of the vanishing points. From this relation it is possible to compute the camera focal length. This relation can be interpreted as the equation of the line orthogonal to a vanishing point, ie, the image of the line at infinity orthogonal to a point at infinity. This is the intersection of the image plane with the plane passing through the camera center and normal to the direction of the vanishing point. One can construct the two lines orthogonal to the two vanishing points and their intersection determines a third vanishing point that corresponds to the direction perpendicular to the landmark plane. So far all the computation involves a single image, and does not require a stereo pair. It is worth noticing that this procedure gives a metric reconstruction of the plane of the landmark in $3 \mathrm{D}$, ie, it allows to perform measures (distances and angles) between geometrical elements on this plane. Furthermore it is possible to compute the $3 \mathrm{D}$ distance $\mathrm{D}$, between the camera (center $\mathrm{O}$ ) and the landmark corner (where $\mathrm{L}$ is the side length of the landmark) as:

$$
\mathrm{D}=\mathrm{L} * \sin \delta / \sin \alpha
$$




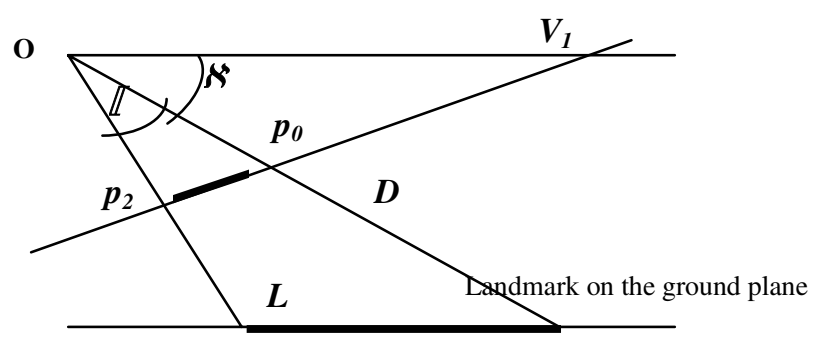

Fig. 2. Correspondence between image plane and landmark direction in the scene

The metric reconstruction with a single image extends to the third dimension (the "vertical") if the angle between the landmark sides is known. However, with a single image, there is not enough information to measure distances in the vertical direction because there is no way to determine the position of an imaged point in space, ie, its projection on the landmark plane. This information is provided by a stereo pair of images. We have two triads of orthogonal vanishing points, one in each camera frame, that correspond to the same triad of orthogonal directions in 3D. These vectors completely define the 3D rotation between the two camera frames, i.e. the homography of the plane at infinity. It is well known that the geometry of a stereo pair of images is subject to the epipolar constraint. A point in one image corresponds to a line of points in the world, and these points project on the second image in a line. The epipolar constraint says that this line must pass through a special point, the epipole, which is the projection of the first camera center in the second image. The stereo correspondence of the vanishing points and the 3D distance of the landmark corner from the two optical centers $\left(D_{1}, D_{2}\right)$ allows to compute the baseline, i.e., the distance between the two camera centers in the world, as shown in Fig 3.

$$
\mathrm{B}^{2}=\mathrm{D}_{1}^{2}+\mathrm{D}_{2}^{2}-2 \mathrm{D}_{1} \mathrm{D}_{2} \cos \left(\beta_{12}\right)
$$

$\beta_{12}$ is the angle between $P_{2}$ and $P_{2 \infty}$, i.e. the result of rotation $\mathbf{R} \times \mathrm{P}_{1}$ in eq (3). Furthermore, the stereo pair allows a more robust calibration by minimizing the error of the stereo geometry between the two images. Keeping fixed the lines of the landmark sides (in the two images), the geometry is determined by the two vertical vanishing points and by the focal length. In fact the vertical vanishing point defines the "horizon" (line at infinity) which intersects the landmark sides in the other two vanishing points. Since the landmark sides are corresponding lines in the two images, these vanishing points are also in correspondence.

The error function to minimize consists of the following geometrical (metric and projective) constraints:

a the aspect-ratio error: i.e. the ratio of the distances between the camera center and the landmark corner computed using the two landmark side (with the single image metric);

the orthogonality error: how far the two vanishing points on the horizon are from orthogonality (the abs cosine of their angle); 
a the distance error: how much the measures of the two landmark sides differs from 1 meter (in the stereo reconstruction);

a the epipolar error: how much any three epipolar lines fail to pass through a single point (the epipole).

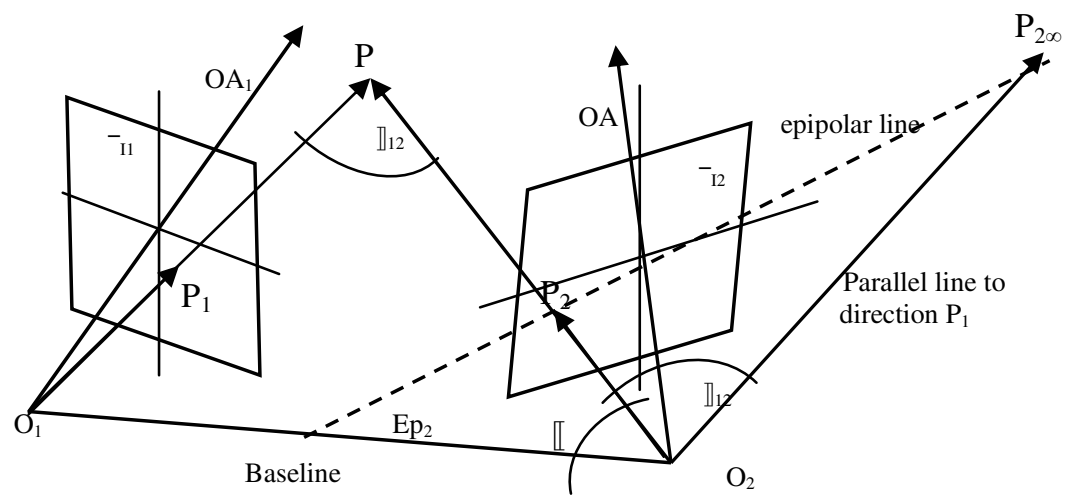

Fig. 3. Binocular stereo configuration

\section{Implementation}

The reconstruction approach described in the previous section has been implemented in an industrial product, for the reconstruction of the ground map of the scene in car accidents. Digital pictures of the scene are already taken by the police personnel for documentation purposes. Therefore our proposed solution, with the minor overhead of placing the reference landmark in the scene, saves the time needed for carrying on and annotating all measures on the spot, deferring this operation to a later time, in the police office. The landmark has been specially designed for easy transport (foldable) and is provided with cylinders covered with a highly reflective film, in order to ensure their visibility in every environment condition when shot by the camera flash. There are three cylinders on the short sides, besides the two at the corners, and one at the midpoint of the hypotenuse, for a total of ten cylinders. The ten cylinders form the basic element and are used to perform the stereo calibration. The sides of the landmark are low-reflecting and black, to improve the automatic detection of the cylinders. The operating procedure starts with outdoor shooting of the scene, taking a series of snaps from different points of view. A standard digital camera is used (4Mpixel) but higher resolution cameras can also be adopted, to improve reconstruction precision.

The operative constraints for the image acquisition are rather simple:

a the landmark should be positioned in the middle of the scene, not too far from the camera;

- to achieve better results, the zoom of the camera should not be changed between the shots using the same focal length, an additional constraint is applied). 
The second step of the operating procedure takes place at office, where the user can run on his own PC a software tool that implements the metric reconstruction of the scene. The application software has been developed under Windows 2000/XP operating systems, with Microsoft Visual C++.
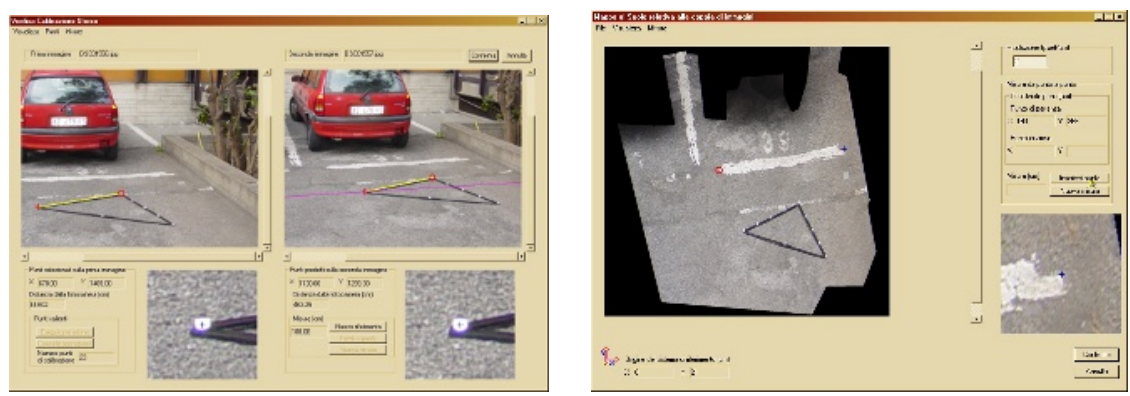

Fig. 4. a) The human interface of LSV (Landmark Stereo Vision); b) ground map

The developed human interface (an example is shown in fig.4) allows the user

- to navigate in the file-system to select image pairs, with optional thumbnail preview;

- to run landmark localization on the images through an automatic procedure (with possible fallback on a manual procedure in case of failure);

$\square$ to define the ground region of interest for each image;

to identify points in stereo correspondence in the image pair and store them in the system;

- to show and print the map of the ground either for each single image or for both of them together;

- to add on the ground map graphic features and text of interest, like point labels;

to perform measures between points, both working on the pair of images, and on the ground map;

- to perform reporting tasks of the measures taken during a work session;

a to save and store all the information in a database, for retrieval and use in a later session of work.

\section{Localization of the landmark}

As one might expect it turns out that a precise localization of the landmark cylinders on the images is crucial for an accurate stereo calibration. In fact this is the only necessary information to start stereo measurement. We use reflecting cylinders and all stereo pictures must be acquired with the camera flashlight on. In this way in any environmental conditions (day and night) the landmark features will appear as bright spots in the images and their detection is greatly simplified. The automatic localization of the cylinders is performed in two steps. The first step roughly localizes the positions using the high luminance level of the cylinders, by thresholding the image intensity and searching for ten blobs of suitable size ( $\mathrm{nr}$ of pixels) and shape (roughly round). The threshold is selected automatically within a selected search area. The second step provides a sub-pixel localization through a correlation with a template model at different scales. Geometric consistency is checked to validate the 
correct detection of such features. There are nevertheless situations where the automatic localization cannot succeed, e.g., when the landmark is placed on a reflecting surface (e.g. a wet road). This ambiguous situation is automatically detected by the process and the user is prompted to manually select and define the locations of the cylinders.

\section{Calibration and ground map reconstruction}

Stereo calibration can be computed using only the landmark points localized on the two images. Nevertheless, it is possible to improve and refine calibration with additional stereo pairs. Such additional points can be easily inserted and edited by the user on the application console. After calibration, the user can perform measures between points selected on the images. The ground region is a polygonal area and it is used in the construction of the ground map. The user may define the ground area by selecting the corners of any polygonal shape on the image, to remove the objects not lying on the floor. The ground map provides a top view of the scene, by merging the information from both image views. It is possible to perform measures on the ground map between manually selected points. It is also possible to project onto this map all measured $3 \mathrm{D}$ points to achieve a full representation of the imaged scene.

\section{Results of Performance}

The procedure has been tested with a set of image stereo pairs by comparing the distances measured by the LSV application software against those measured on the scene with a plastic metric tape. The reference distances are therefore affected by an error of $1 \%$ (typical error of measures with a plastic metric tape).

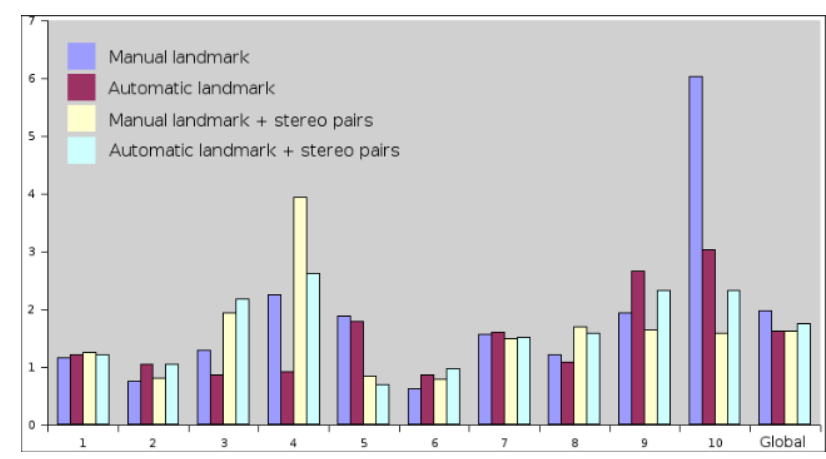

Fig. 5. 3D measurement error for 20 different stereo pairs

As we said the reconstruction depends strongly on the location of the feature elements. Fig.6 shows the average percentage measure errors of a few image pairs for different configurations of stereo calibration. Red-bar refers to measurements with automatic detection of landmark cylinder features; white bar is the accuracy by using automatic detection + additional stereo matched points (user selected). Blue-bar refers to manual selection of cylinders (light-green-bar corresponds to manual selection + stereo matched points). Overall the accuracy is better than $98 \%$; only a few times the 
measurement error is higher than $2 \%$. Although this is twice that of a tape, it has been proved sufficient in most practical situations. Clearly the accuracy of the measures is very accurate around the landmark and decreases for points far from it. There is almost no difference between the automatic location of cylinders and the manual location. In general the inclusion in stereo calibration of stereo pairs manually selected on the images does not improve the result significantly. However it is useful when the localization of the cylinders cannot be accurate enough.

\section{Conclusions}

The paper describes an industrial application of Landmark-based Stereo-Vision by using the vanishing point representation. To achieve a robust implementation our approach relies on the presence of an artificial landmark having an orthogonal triangular shape with sides of one meter length. Reflecting cylinders are used to allow an easy detection and location of landmark features, since both stereo images are acquired using a flash light (night and day conditions). The proposed systems is currently tested for the reconstruction of car-accident scenes by police operators, using a standard un-calibrated photo-camera, without any additional constraint for the user. The application software provides a simple and user-friendly interface to perform all image analysis and 3D measurement in a back-office environment. The achieved performance shows a measurement error that is always better than $2 \%$ (with a 4Megapixel camera). Further research activity is in progress to achieve significant improvements in self-calibration and in multi-view integration.

\section{Acknowledgements}

Many thanks go to Biancamaria Zerbinati for her valuable support in the software development of the system integration environment and the Graphical User interface that has been greatly appreciated by the users for its effectiveness.

\section{References}

1. Real-Viz Image-Modeler, http://www.realviz.com/products/im/index.php

2. R.Cipolla, D.P.Robertson, E.G.Boyer, "Photobuilder-3 Models of Architectural Scenes from Uncalibrated Images", proc. IEEE Int. Conf Multimedia Computing and Systems ICMCS'99, vol. 1, pp. 25-31, 1999.

3. B.Galvin, "Photogrammetry: fast crime-scene measuring with close-range photogrammetry", Evidence Tech. Magazine, Sep.2004, www.EvidenceMagazine.com.

4. G.Garibotto, M.Corvi, C.Cibei, S.Sciarrino, "3D-MODS, Moving Obstacle Detection System”, Proc. Int. Conf on Image Analysis \& Processing, ICIAP’03, Mantova, 2003.

5. O.D.Faugeras, “Three-Dimensional Computer Vision, A geometrical viewpoint, MIT press, Cambridge, MA, 1996.

6. PHS.Torr, DW.Murray Int J. Computer Vision 24 271-300 (1997).

7. E.K.Bas, J.D.Crisman, “An easy to installa camera calibration for traffic monitoring”, Proc. Of IEEE Conf. On Intelligent Transportation Systems, 1997, pp. 362-366.

8. B.Caprile, V.Torre, "Using Vanishing Points for Camera Calibration", Int.J.Computer Vision, vol. 4, n.2, pp. 127-140, 1990. 